\title{
Erratum to: Arithmetical approach to the twin primes conjecture
}

\section{E. Benedetto}

\section{Erratum to: Ann Univ Ferrara DOI 10.1007/s11565-009-0070-8}

\section{Conclusion}

The line 1 is not "Twin primes problem is well know in number theory and during the last two centuries many mathematicians have attempted to solve this problem using different methods" but "In this paper, we have considered an arithmetical approach to the Eratosthenes sieve and to the problem of the twin primes. The objective was only to introduce an elementary approach to the study of"

The line 2 is not "In this paper starting from the partition of natural number and by considering four selection rules, we analyzed the arithmetical sieve of prime numbers and arithmetical properties of the twin couples." but "prime numbers, but it was not a demonstration of the twin prime conjecture. In the lastline of the Sect. 4 there is an error since "Therefore there are infinitely many twin primes" is not proved."

The online version of the original article can be found under doi:10.1007/s11565-009-0070-8.

\footnotetext{
E. Benedetto $(\varangle)$

University of Salerno, Salerno, Italy

e-mail: elmobenedetto@libero.it
} 